\title{
Plasma dynamics of a prominence associated coronal mass ejection
}

\author{
D. Tripathi ${ }^{1}$, V. Bothmer ${ }^{1}$, S. K. Solanki ${ }^{1}$, R. Schwenn ${ }^{1}$, \\ M. Mierla ${ }^{1}$, G. Stenborg ${ }^{2,3}$ \\ ${ }^{1}$ Max-Planck-Institut für Sonnensystemforschung, 37191 Katlenburg-Lindau, Germany \\ email: [tripathi; bothmer; solanki; schwenn; mierla@linmpi.mpg.de] \\ ${ }^{2}$ NASA Goddard Space Flight Center, Greenbelt, USA \\ ${ }^{3}$ Catholic University of America, USA email:stenborg@kreutz.nascom.nasa.gov
}

\begin{abstract}
An erupting prominence seen by SOHO/EIT was tracked into the field of view of the LASCO C2 and C3 coronagraphs where it developed into the core of a structured CME. The erupting prominence was deflected by an angle of $\sim 20^{\circ}$ towards the north pole whereas the consequent core of the CME and it's leading edge propagated in the outer corona at constant position angle. The prominence material underwent a constant acceleration phase until a height of $\sim 1.5$ solar radii before it started to decelerate up to a distance of 5.0 solar radii. An inflow of plasma with a speed of about $70-80 \mathrm{~km} / \mathrm{s}$ was discovered in the EIT observations at a height of 1.5-1.2 solar radii in the course of the prominence eruption, matching in time the prominence deceleration phase. The downflowing material followed a curved path, suggestive of the apex of a contracting magnetic loop sliding down along other field lines.
\end{abstract}

\section{Introduction}

Detection of sunward moving plasma after CME eruptions has recently been reported by several authors (Innes et al. 2003 and references therein). These downflows were interpreted as shrinking of magnetic field lines that had opened during the solar eruptions. Based on the complementary observations of TRACE and RHESSI, Asai et al. (2004) interpreted the nonthermal radiation detected at times of the downflows as evidence for magnetic reconnection processes underneath the CME. In this study we report a downflow of plasma identified in EIT observations taken at $195 \AA$ in the course of a prominence associated coronal mass ejection.

\section{Observations and Conclusions}

(a) The downflow of the plasma occured approximately 90 minutes after the onset of the prominence eruption at a height of about $1.5 R_{\odot}$ as seen by EIT at $195 \AA$ (Figure 1 ). The speed of the inflow was about $70-80 \mathrm{~km} / \mathrm{s}$. There was no evidence for any inflow in the coronagraphic images taken by LASCO/C2 \& C3.

(b) A deflection of about 20 degrees towards the north pole was observed in the early phase of the prominence eruption (Figure 2 left panel). Height-time analysis of different specific features along the prominence showed similar position angle, height-time and speed-height profiles.

(c) The height-time profiles revealed that the downflow started at times of the deceleration phase of the CME core (Figure 2 right panel).

(d) The leading edge of the CME observed in the LASCO/C2 \& C3 FOV propagated with a speed of $860 \mathrm{~km} / \mathrm{s}$ whereas the speed of the CME core was just $210 \mathrm{~km} / \mathrm{s}$ (Figure 2 right panel). 

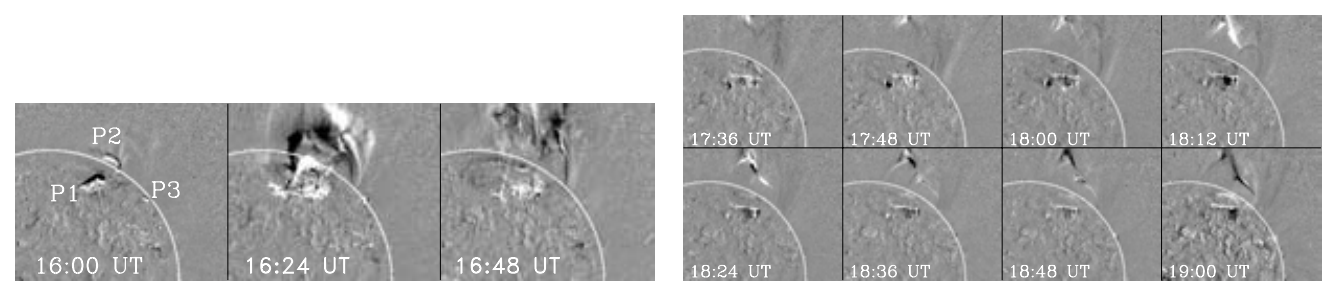

Figure 1. Left Panel: Running difference images taken by EIT at $195 \AA$ showing simultaneous eruptions of the prominences marked by P1, P2 and P3. The eruption started on 05/03/00 at around 16:00 UT. In all images North is up and West is top the right. Right Panel: Series of EIT $195 \AA$ running difference images displaying the downflow after the eruption. The dowflow, visible as the bright feature in the NW part of the limb, started at around 17:36 UT in the EIT FOV.
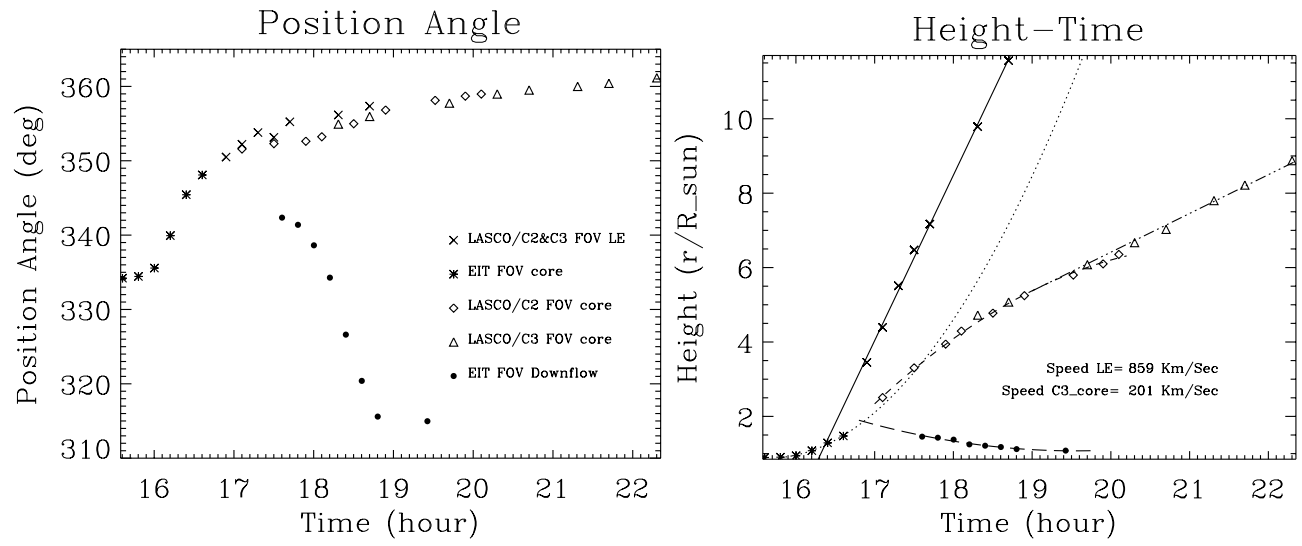

Figure 2. Left panel: Position angle variation with time of prominence in the EIT FOV (asterisks) and CME in LASCO/C2 (diamonds) and in C3 (triangles) FOV. The PA variation with time of the leading edge and the inflow in the EIT FOV are presented by crosses and solid dots respectively. Right Panel: Height-time profile for the prominence (asterisks), the core in LASCO/C2 (diamonds) \& C3 (triangles), the leading edge (crosses) and the inflow (solid dots).

The results emphasize the importance of further studies of the height-time evolution of prominences in the context of the internal structures of CMEs from the low corona up to several solar radii distance from the limb.

\section{Acknowledgements}

DT is thankful to Dr. B. Inhester, Dr. N. -E. Raouafi for several usefull discussions and to IMPRS for providing the funding to attend this meeting. This study is part of the scientific investigations of the project Stereo/Corona supported by the German "Bundesministerium für Bildung und Forschung" through the "Deutsche Zentrum für Luft- und Raumfahrt e.V." (DLR, German Space Agency) under project number 50 OC 0005. We also thank the SOHO/LASCO/EIT consortium for providing the data.

\section{References}

Asai, A; Yokoyama, T; Shimojo, M.; Shibata, K. 2004 Astrophy. J. 605, L77-L80.

Innes, D. E.; McKenzie, D. E.; Wang, T. 2003 Sol. Phys. 217, 247-265. 